\section{Perception is far from perfection: The role of the brain and mind in constructing realities}

\section{Itiel E. Dror}

School of Psychology, University of Southampton, Southampton, S017 1BJ, United Kingdom. id@ecs.soton.ac.uk http://www.ecs.soton.ac.uk/ id

Abstract: Dichotomizing perceptions, by those that have an objective reality and those that do not, is rejected. Perceptions are suggested to fall along a multidimensional continuum in which neither end is totally "pure." At the extreme ends, perceptions neither have an objective reality without some subjectivity, nor, at the other end, even as hallucinations, are they totally dissociated from reality.

Higher-level visual cognition is achieved through complex and dynamic interactions, both within the many processing components of the visual system and between the visual system and other brain areas and systems. Before considering the multiplicity of factors that play a role in determining what we see and experience, it is important to pull back and consider one's basic conceptualization and theoretical outlook. Such philosophical issues have (implicitly, if not explicitly) implications for how we approach and conduct research, and even for the very questions we ask and the type of answers we seek (Dror \& Dascal 1997; Dror \& Thomas 2005).

A naïve view of visual cognition deems that it normally provides perceptions and experiences that have an objective reality. Failures to see things correctly, such as in hallucinations, need to be investigated and explained as system malfunctions. This framework dichotomizes perceptions as either "normal" (i.e., having an objective reality) or as "failures" (i.e., lacking an objective reality). Other perceptual problems, such as illusions, misidentifications, distortions, and misperceptions, may be considered as a subcategory in which perceptions "fail" yet stem from an "objective reality" rather than being totally dissociated from it.

The nature of perception. An alternative view is suggested in which perceptions are seen to fall along a multidimensional continuum. Even at the far extremes of the continuum, perceptions are not entirely "pure." At the one extreme, perceptions never have a full and total "objective reality," and at the other extreme end, hallucinations and delusions are not totally dissociated from reality.

The underpinning of this framework is not its continuum nature, but that perception is never totally objective to begin with. The lack of objective reality across the plurality of perceptions results in a multidimensional continuum. If one adopts this view, then rather then asking what is wrong or wondering why the system is failing, one tries to understand what factors mediate perception and how they interact. Perhaps the former outlook derives from a more clinical approach and perspective whereby some people are considered "normal" and hence perceive the "objective reality," whereas patients and clinical populations have "disorders" because of system failures to have an "objective reality."

Understanding that perception is far from perfection provides a contextual framework for examining the visual cognitive system. The mind and the brain are dynamic systems that play active roles in how we perceive and construct realities. Our perceptions depend on a whole range of factors, which I will try to illustrate.

Mental states. Mental states play a critical role in how perceptual information is processed. For example, our hopes, fears, and expectations affect what we perceive. In a recent laboratory experiment, emotional states were shown to affect whether two visual patterns were perceived as the same or as being different (Dror et al. 2005). There are numerous phenomena that can further illustrate how the mind plays an active role in how we perceive and construct reality, such as motivation, wishful thinking, cognitive dissonance, self-fulfilling prophecies, and confirmation bias (e.g., Darley \& Gross 1983; Festinger \& Carlsmith 1959; Snyder et al. 1977).

Cognitive factors. Visual cognition is a set of complex and interactive processes (e.g., Grill-Spector et al. 1998). No cognitive system works on its very own. Each cognitive system is intertwined and interacts with a range of other cognitive systems. For example, how information is understood, processed, and collected depends on how it compares against information already stored in memory (e.g., Kosslyn et al. 1994). The influence of such processing further depends on how it is represented, available resources, goals of the system, context, and other factors (e.g., Eberhardt et al. 2004; Maier 1930; Reuter-Lorenz 2002; Smith \& Dror 2001).

Perceptual mechanisms. Even the lower-level sensory mechanisms, which initially perceive and encode the input to the system, are not passive or isolated from a variety of factors. They try to make (impose) sense and consistency on the world around us, even when the input presents ambiguous or impossible information (e.g., Dror et al. 1997). Among other things, the perceptual mechanisms adjust and change sensitivity thresholds, segment and chunk information in a variety of ways, and perceive colour and lighting based on their own parameters settings (e.g., Land 1964; Prinzmetal 1995). Therefore, much of what we perceive, even at the lower-level mechanisms, is dependent on the perceiver rather than reflecting objective reality.

Bottom-up, top-down, and mental imagery. Perception and cognition, at all their levels, depend on bottom-up, data-driven processes and on top-down, conceptually driven processes (e.g., Humphreys et al. 1997). The top-down processes may be viewed as the source of subjectivity, individualization of perception, and distancing from the "objective reality." However, even the sensory mechanisms in a purely bottom-up mode do not reflect reality as it "really" is.

Mental imagery is a range of phenomena where perception and experience occur without direct perceptual input. Positron emission tomography (PET) and other studies have demonstrated that the same brain substrates are used to process imagined and perceived images, except that in imagery the input comes from other cognitive systems (Kosslyn et al. 1993; 1997). Furthermore, visual mental rotation, for example, shows that imagination follows the laws of physics and rotations in the physical world (e.g., Smith \& Dror 2001). Hence, although all the processes involving imagery do not have direct input from the external world, the input and the way it is processed is not dissociated from normal perception.

Summary and conclusions. We are different people, with different experiences, different views, and different brains and sensory mechanisms. This entails that we have different perceptions. Most people share sufficient perceptual commonalities that allow labelling and communication within everyday life activities. Nevertheless, the perceptions across people are far from identical. Furthermore, even if we did perceive the exact same thing, that percept is not necessarily a true and accurate reflection of the "objective reality."

Perceptions fall along a multidimensional continuum and are subjective in nature. This individualization of perception derives from the active nature of cognition and the wide range of factors that affect what and how we perceive. 